\title{
System approach to AAL applications: a case study
}

\author{
Lenka Lhotska, Jan Havlik, and Petr Panyrek \\ 1 Department of Cybernetics, Faculty of Electrical Engineering \\ Czech Technical University in Prague, Technická 2, CZ-16627 Prague 6 \\ ${ }^{2}$ Department of Circuit Theory, Faculty of Electrical Engineering \\ Czech Technical University in Prague, Technická 2, CZ-16627 Prague 6 \\ ${ }^{3}$ HIGH TECH PARK, Rašínovo nábřeží 56, CZ-12800 Prague 2 \\ lhotska@fel.cvut.cz, xhavlikj@fel.cvut.cz, \\ Petr.Panyrek@combitrading.cz
}

\begin{abstract}
The objective of AAL and home care is a better care for frail individuals (elderly chronic and disabled patients) in a home care setting. To improve this kind of care means to allow the citizens to stay at home as long as possible, delaying the institutionalization of people, possibly avoiding it for a high percentage of them. Recent development in ICT shows that it is almost impossible to design and implement an AAL system as fixed to certain hardware, operating system, and infrastructure. Thus it is necessary to develop such architectures that will be easily extensible and modifiable. We will discuss such approaches in the paper.
\end{abstract}

Keywords: assistive technologies, HomeBrain, health monitoring, ambient assisted living

\section{Introduction}

The objective of AAL and home care is a better care for frail individuals (elderly chronic and disabled patients) in a home care setting. To improve this kind of care means to allow the citizens to stay at home as long as possible, delaying the institutionalization of people, possibly avoiding it for a high percentage of them. Institutionalized elderly citizens are at high risk of cognitive impairment, functional loss, social isolation, or death. $[6,7]$

Integrating information deriving from different sources and implementing it with knowledge discovery techniques allows medical and social actions to be appropriately performed with reliable information, in order to improve quality of life of patients and care-givers. To stay at home means to keep independency, self-sufficiency, social network role.

Currently the mobile technologies, sensors and other devices enable collecting vast amount of data of individuals. This multi-parametric data may include physiological measurements, genetic data, medical images, laboratory examinations and other measurements related to a person's activity, lifestyle and sur- 
rounding environment. There will be increased demand on processing and interpreting such data for accurate alerting and signalling of risks and for supporting healthcare professionals in their decision making, informing family members, and the person himself/herself.

Recent development in ICT shows that it is almost impossible to design and implement an AAL system as fixed to certain hardware, operating system, and infrastructure. Thus it is necessary to develop such architectures that will be easily extensible and modifiable. We will discuss examples of such approach in next sections together with our suggestion of integration of the topic into educational process.

\section{Motivation}

The main idea is to design and develop an integrated platform (both hardware and software) that would help people especially in their home environment when they are suffering from certain disabilities or have to perform rehabilitation after an injury or brain stroke or "only" help increase their well-being.

Till now most of the solutions are represented by isolated tools, devices or pieces of software. But many of the health problems are rather complex and thus need complex approach to assistance.

For example, a person after a brain stroke has certain motoric problems and also speech problems. It is necessary to perform rehabilitation in both directions and also evaluate results jointly. The rehabilitation process must be monitored as objectively as possible. That means using defined measurement and evaluation of measured data. It is difficult to estimate progress of rehabilitation only from subjective observations. Training of motoric functions can be monitored by cameras, sensors (e.g. accelerators, pressure sensors). From these signals and images we can evaluate motion range, ability to open and close fist, walking, etc. And we can evaluate temporal development. Similarly we can approach the problem with speech. The person can utter sentences and speech recognition system analyses and classifies quality of the utterance. Again we can evaluate temporal development and see whether there is any improvement.

Similar scenarios can be applied to other health problems or disabilities.

The point is to integrate finally all information from partial processes and evaluate the complete state of the person. That can help both the clinicians for focusing their effort and also the patient for finding motivating solution (to intensify activity where the progress is slower). [1-4]

\subsection{General System Design}

The system should be realized as a hierarchical distributed system utilizing flexible software platform, as for example multi-agent architecture. Collected data and signals should be stored locally in a data repository; defined part of the data and aggregated information should be send to a clinical data storage (could be part of a hospital information systm, depends on the concrete solution). The 
HL7 standards will be followed. In data and signal evaluation advanced methods known from signal processing and data mining fields will be used. It is highly advisable to use defined standard on all levels of data storage, communication, and processing.

One of the key issues is also proper design of sensor network necessary for corresponding data collection. The network should be optimized, i.e. using minimum set for acquisition of maximum relevant data according to the diagnosis and therapy of the concrete person. [8]

\subsection{New Technologies Closer to People}

The 21st century brings many new technological achievements into our lives. However at the same time there are many challenges connected with high-tech. Quite frequently they appear on the social level - many potential users are not prepared to become the users. Not everybody is technically skilled and not everybody wants to read long and complicated manuals that are sometimes written in a very difficult style (even for technically educated people). Having in mind that the potential users may recruit from senior or handicapped population the user interface must be adapted to their needs.

One of the most significant achievements is definitely the internet, as a breakpoint in communication platforms. It provides an infrastructure for various technologies that can dramatically improve quality of our lives and make lives more pleasant. Imagine a device that would perform many needed household tasks, monitor our health state and thus become a "home brain" for us. This idea has been behind the project described as an example of integrating platform in the Section 4 .

\section{Centre of Assistive Technologies project}

We have established a specialized facility - Centre of Assistive Technologies composed of several laboratories and working places: smart home, medical technology and biosignal laboratory, audio/video media laboratory, communication laboratory, and computer/seminar room. The first two are the core of the centre, the remaining three serve for education and research in connected areas and provide also support for the main part. The medical technology and biosignal laboratory has three sections, namely fully equipped intensive care unit (ICU), EEG laboratory (where also other biosignal measuring devices are placed) and medical technology section. This laboratory is focused on research and education that is closely linked with medicine. The intention is to introduce to students most of the technology they can meet in hospitals and in some cases in home environment. They have to understand the processes from the technical point of view. For example, at ICU there is also complete installation of electric power and data networks as in hospitals because the hospital installations have to satisfy more strict requirements concerning electric safety. Here we add new sensors that allow additional monitoring of the patient with respect to his/her safety, state 
of the bed, etc. The main idea is to design and develop approaches and successively devices that will be able to indicate certain medical problems, one of them indicated by medical doctors is hydration of the patient. Currently only indirect evaluation is done, namely volume of urine. In this area also research connected with electromagnetic compatibility, interference of wireless communication with medical devices will be performed.

The smart home is a test bed for design and development of different setups and functions that can be finally used in inhabited homes. The basic functions currently prepared are: security, control of home appliances, monitoring persons activity, and monitoring persons health state. Now we describe individual functions and technology behind. Security means protection against intruders and we add also safety in the environment. For the security function we can use cameras, PIR-based motion detectors, detectors of open/closed windows and doors. Safety function should control whether specified devices and home equipment are in required state, e.g. whether iron or cooker are switched off when leaving the home, or whether the water tap is closed. Monitoring persons daily activity is important when the person lives alone and has certain disabilities. Then we have to check whether the person is doing well, has not fallen down and injured. Another case is prescribed physical activity or rehabilitation, e.g. after an injury, brain stroke or when multiple sclerosis is diagnosed. Here we can make use of sensors installed for security function and additionally accelerometers, tactile sensors, etc. Monitoring persons health state can make use of the knowledge and skills acquired in the medical technology and biosignal laboratory. Only for personal monitoring we need miniaturized devices. Again according to the diagnosed health state we can propose corresponding setup of measuring equipment (e.g. ECG, blood sugar, blood pressure, body temperature, transpiration, breathing). Recent medical studies have shown that especially in elderly population there is problem with food and liquid intake. So one of the challenges is to monitor unobtrusively and relatively precisely how well the person is nourished. On the

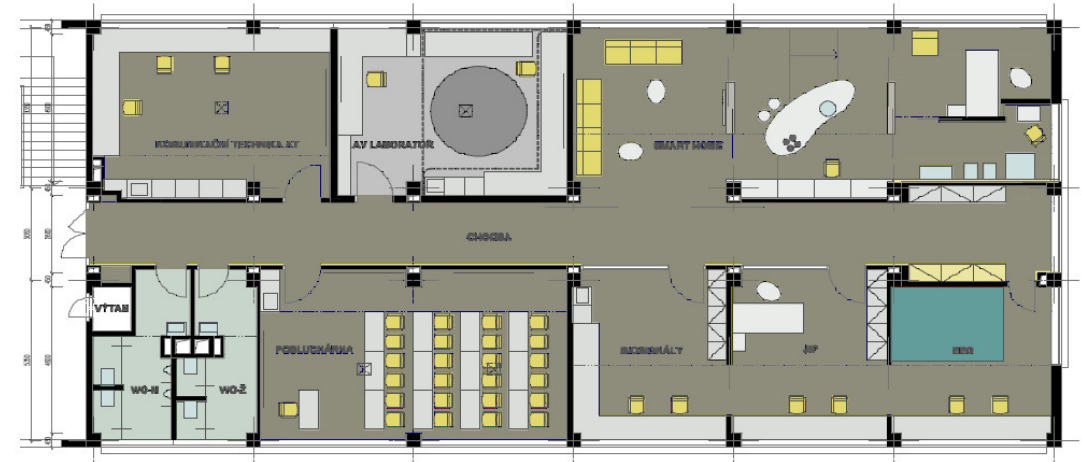

Fig. 1. Ground plan of CAT 
other hand at obese people it would be welcome to monitor amount of food and provide recommendation and warning against excessive food intake.

The facility will serve for practical project-based education of students in assistive technologies (AT). The situation in the given field may be characterized in following way: there are no graduates specialized in AT; there is no complex educational program in AT; there exist a number of information barriers between disciplines that may be solved exclusively by consistent interdisciplinary integration; there exists social and objective demand for employees in AT; in the Czech Republic there are relatively many SMEs and institutions focused on AT that need graduates and support for life long learning of current employees. The courses will become a core of an interdisciplinary study branch in Master study and at the same time they will be offered in life long learning. The core courses cover the following topics: data sensing and transmission; sensors, security and control in assistive environment; circuitry and system principles of electronic devices for AT; advanced methods of data mining and knowledge discovery and their applications in AT; telecommunication equipment and systems for AT; multimedia technology. The topics are systematically and logically interconnected. The content is designed in such a way that theory, applications and system integration are represented in suitable proportion.

The Centre will serve as a platform both for education and for applied research in the area of AAL. The main idea is to offer space for development of integrating solutions. Currently there are many partial solutions of smart homes, home security, health state monitoring, internet services. The problem is that these applications have been developed separately and usually they work as closed systems without possibility to interconnect them. The aim is to offer a standard user interface and communication for interconnection of various services and devices.

\section{Case study: HomeBrain - TV Computer}

A new project has been started by a Czech company High Tech Park in cooperation with several other companies. The main idea is to integrate many different functions and unify the control interface. Actually the user interface is the core of the project with the aim to have the system control as simple and intuitive as possible. A TV computer serves as the user interface and can be easily controlled in the same way as a standard TV set. The authors have named the project HomeBrain. It fulfils several main functions, namely gate to the internet, multimedia services, senior monitoring, health state monitoring, social networking of HomeBrain users, remote control of home devices, intelligent security system. Additionally, it can be connected to telebanking, e-services (including e-government), and other electronic services. HomeBrain is an example of an integration platform that provides the user with a relatively simple interface. It can be easily extended by newly developed modules. This feature also means that the configuration can be designed on demand based on the user 
requirements because not everybody wants to have complete set of all possible functions and installed devices. [5,9]

The designers have defined several basic requirements on the HomeBrain. It must be small, having the size of a common TV set-top box and being easily controllable without any need to study a long and complex manual. The HomeBrain device installation is simple - it only needs to be connected to the TV set and internet. Thanks to the new Atom processor (Intel company) the HomeBrain can offer very good technical characteristics. Microsoft environment is used for development of applications and interconnection with other technologies.

Let us focus now on the typical functionalities of the HomeBrain system. In addition to classic TV programs it is possible to watch programs on Internet TV. Search for selected music can be easier - no need to try to find a particular CD or

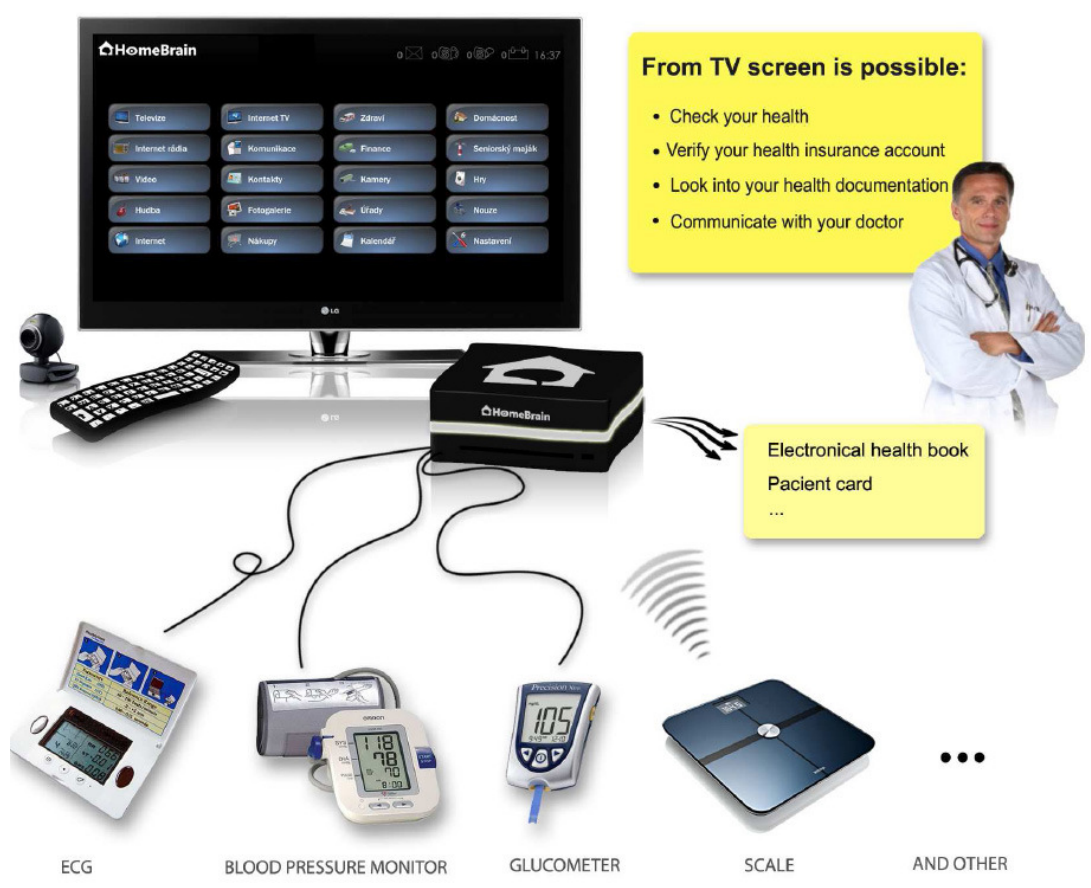

Personal Medical Monitoring Devices
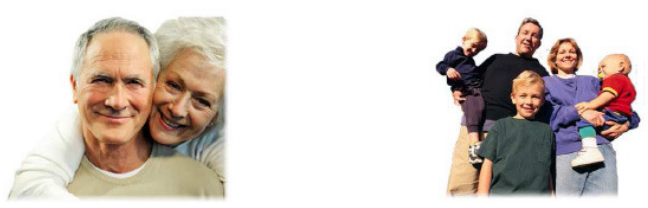

Fig. 2. HomeBrain - TV computer 
DVD in boxes or stands. Music can be downloaded from the internet (using legal way), or from a storage on the hard disk and stored in the catalogue. Movies can be lend in a virtual rental store. Browsing digital photographs, creating albums and sharing them with friends is another pre-defined function.

If the user wants to control home appliances remotely on the TV, phone, or via internet the system is easily extensible. The only condition is that the home appliances have the option of remote control. Another possible function is intelligent security. The user interface is interconnected with the installed security system and allows intelligent evaluation.

Eshop is another option. It can be comfortable for elderly people, for people with motoric disabilities or even for ill people. There is a special service that uses bar code reader. Thus regularly bought food can be easily ordered again. The information is sent from the reader to the HomeBrain and then the list of required items is sent to the particular eshop. Of course, necessary condition is existence of such a shop in relatively close distance.

Health state monitoring is becoming more and more important area of AAL applications. Heart-attacks, diabetes, increased cholesterol are typical manifestations of unhealthy lifestyle at present time. Timely anticipation of such state can save lives. Another group is represented by chronically ill persons who must be regularly checked (or even monitored over longer time span). There have been developed many different applications using various sensors and devices for measurement of physiological values. The HomeBrain has an interface for data transfer from such sensors or devices. The user can decide whether the health state information is only stored locally or also sent to his/her family doctor.

The system also offers a similar function to Skype, namely remote contact with other family members or friends living in distant places. There are more functions ready, i.e. calendar, e-mail, video calls, sms messaging, telebanking, eservices (e.g. contact with local authorities), monitoring elderly family members remotely, internet browsing, games. Functions that depend on external services can be active only if the services are available at the given location. This concerns, for example, delivery of food ordered in the eshop, electronic contact to local authorities.

\section{Conclusion}

We have presented an example of integration platform that allows adding new functional modules in "plug-and-play" manner. An important issue concerns data and its format for storage and communication. Many existing systems are not interoperable because they use proprietary formats and not any existing standard. In such applications as AAL systems that assume gradual extension and adding of new components represents standardization very important requirement. We also believe that it is necessary to introduce all these topics in engineering education. Thus we have designed project- and problem-based education in assistive technologies. A new facility - Centre of Assistive Technologies - has been established. It will serve for students work and application research projects. 


\section{Acknowledgement}

This work has been supported by the ENIAC JU Project MAS "Nanoelectronics for Mobile Ambient Assisted Living (AAL) Systems" and the research program No. MSM 6840770012 of the Czech Technical University in Prague (sponsored by the Ministry of Education, Youth and Sports of the Czech Republic).

\section{References}

1. Celler, B.G., Earnshaw, W., Ilsar, E.D., Betbeder-Matibet, L., Harris, M.F., Clark, R., Hesketh, T., Lovell, N.H.: Remote monitoring of health status of the elderly at home. A multidisciplinary project on aging at the university of new south wales. International journal of bio-medical computing 40(2), 147-155 (10 1995)

2. Chan, M., Esteve, D., Escriba, C., Campo, E.: A review of smart homes - present state and future challenges. Computer methods and programs in biomedicine 91(1), $55-81$ ( 7 2008)

3. Costin, H., Rotariu, C., Morancea, O., Andtuseac, G., Cehan, V., Felea, V., Alexa, I., Costin, C.: Complex telemonitoring of patients and elderly people for telemedical and homecare services. In: New aspects of biomedical electronics and biomedical informatics. pp. 183-187 (August 20 - 22 2008)

4. Dang, S., Golden, A.G., Cheung, H.S., Roos, B.A.: Telemedicine Applications in Geriatrics, pp. 1064-1069. Brocklehurst's Textbook of Geriatric Medicine and Gerontology, W.B. Saunders, Philadelphia (2010)

5. HIGH TECH PARK: Project3B HomeBrain (2011), http://www.htpark.eu/en/solutions/r1-project3b-homebrain/

6. John, P.D.S., Montgomery, P.R., Kristjansson, B., McDowell, I.: Cognitive scores, even within the normal range, predict death and institutionalization. Age and Ageing 31(5), 373-378 (2002)

7. Molaschi, M., Scarafiotti, C., Chiantelassa, D., Magnano, A., Ferrario, E.: Evaluation of cognitive and behavioral status of institutionalized elderly. follow-up two and seven years. Archives of Gerontology and Geriatrics 26(1), 335-342 (1998)

8. Rialle, V., Lamy, J.B., Noury, N., Bajolle, L.: Telemonitoring of patients at home: a software agent approach. Computer methods and programs in biomedicine 72(3), 257-268 (11 2003)

9. Zdravotnické noviny: New project for elderly in Czech republic, thay will have not to be instituonalized (in Czech) (2011), http://www.zdn.cz/clanek/zdravotnickenoviny/v-cr-se-chysta-projekt-pomoci-seniorum-nebudou-muset-do-ustavu-449873 